\title{
Properties of fast carbon cluster microbeams produced with a tapered capillary
}

\section{AUTHOR(S):}

Tsuchida, Hidetsugu; Tomita, Shigeo; Nishimura, Kazushige; Murakoshi, Ryohei; Naitoh, Masahiro; Sasa, Kimikazu; Ishii, Satoshi; Yogo, Akifumi; Itoh, Akio

\section{CITATION:}

Tsuchida, Hidetsugu ...[et al]. Properties of fast carbon cluster microbeams produced with a tapered capillary. Nuclear Instruments and Methods in Physics Research Section B: Beam Interactions with Materials and Atoms 2012, 293: 6-10

\section{ISSUE DATE:}

2012-12

URL:

http://hdl.handle.net/2433/172451

\section{RIGHT:}

(c) 2012 Elsevier B.V.; この論文は出版社版でありません。引用の際には 出版社版をご確認ご利用ください。; This is not the published version. Please cite only the published version. 


\section{Properties of fast carbon cluster microbeams produced with a tapered capillary}

Hidetsugu Tsuchida ${ }^{1,2 *}$, Shigeo Tomita ${ }^{3}$, Kazushige Nishimura ${ }^{2}$, Ryohei Murakoshi ${ }^{2}$, Masahiro Naitoh $^{2}$, Kimikazu Sasa ${ }^{4}$, Satoshi Ishii ${ }^{4}$, Akifumi Yogo ${ }^{5}$, and Akio Itoh ${ }^{1,2}$

${ }^{1}$ Quantum Science and Engineering Center, Kyoto University, Uji, Kyoto 611-0011, Japan

${ }^{2}$ Department of Nuclear Engineering, Kyoto University, Kyoto 606-8501, Japan

${ }^{3}$ Institute of Applied Physics, University of Tsukuba, Tsukuba, Ibaraki 305-8573, Japan

${ }^{4}$ Tandem Accelerator Complex, University of Tsukuba, Tsukuba, Ibaraki 305-8577, Japan

${ }^{5}$ Photo-Medical Research Center, Japan Atomic Energy Agency, Kyoto 619-0215, Japan

PACS codes: $34.35 .+\mathrm{a}, 37.20 .+\mathrm{j}, 79.77 .+\mathrm{g}$

Keywords: carbon clusters, microbeam, tapered glass capillary optics

\section{Abstract}

We study the transmission properties of fast cluster ions $\left(\mathrm{C}_{n}^{+}(n=2-4)\right.$ with velocities ranging from 0.89 to 1.79 a.u.) through a single tapered capillary with an outlet diameter of $13.7 \mu \mathrm{m}$. We investigate the projectile-velocity dependence of the transmission fraction from the measurements of transmitted particle energy spectra. It is found that the non-fragmentation fraction of $\mathrm{C}_{2}^{+}$projectiles increases with decreasing velocity, indicating that fragmentation occurs mainly via close collisions with the surfaces of the capillary wall. For $\mathrm{C}_{n}^{+}(n=2-4)$ ions with the same incident velocity, the cluster-size dependence of the non-fragmentation fraction shows even-odd alternation, implying that the fraction includes contributions from stable clusters surviving the grazing scattering process at the capillary 
surface. We also find that the angular distribution of transmitted particles is narrower for cluster projectiles compared with atomic projectiles.

\section{Introduction}

Charged particle microbeams are powerful tools for microstructural analysis of materials at micro- and nano-scales, with widespread nanotechnology applications for fabrication, manipulation, and lithography of materials. Recent studies focus on biological applications, such as mapping or imaging of atomic and molecular particles in cellular material. Standard techniques for producing microbeams for radiobiological research employ collimation with pinhole apertures or focusing with electrostatic and/or magnetic lenses. Recent developments for producing charged particle microbeams include a tapered glass capillary with a micron-sized outlet diameter [1-6]. The capillary-microbeam method allows for visual pinpoint-irradiation of a selected target area under in-air environment [7-10].

Compared with standard methods, the capillary microbeam method allows to easily produce focused beams, for various types of charged particles in a wide energy range (keV to $\mathrm{MeV}$ ), without special adjustment or handling. Capillary transmission studies for various types of incident beams, such as slow and fast ions [1-3], fast electrons [4,5], and slow positrons [6], demonstrate that the transmission process depends on the projectile velocity and is independent of the projectile mass. The latter result implies that the capillary method is applicable of producing microbeams of heavy particles such as clusters. The application will lead to the development of new techniques for ion beam material analysis such as secondary ion mass spectrometry (SIMS) or ion beam microfabrication. It is of practical importance to study the beam quality in relation to beam composition (type of transmitted particles), monochromaticity (outgoing energy distribution), and angular distributions (beam spot size). 
In this work, we study the capillary-transmission properties for small-sized cluster projectiles of $\mathrm{C}_{n}^{+}(n=2-4)$ with velocities between 0.89 and 1.79 a.u.. In particular, we investigate energy spectra and beam spot sizes of transmitted particles through the capillary.

\section{Experiment}

Figure 1 shows the experimental arrangement. Carbon clusters from a Cs-sputter ion source were accelerated with the $1 \mathrm{MV}$ Tandetron accelerator at the University of Tsukuba. We employ the following projectile beams: $0.24-0.96 \mathrm{MeV} /$ atom $\mathrm{C}_{2}{ }^{+}$ions, $0.24 \mathrm{MeV} /$ atom $\mathrm{C}_{3}{ }^{+}$ ions, and $0.24 \mathrm{MeV} /$ atom $\mathrm{C}_{4}{ }^{+}$ions. The projectile beams were collimated by two sets of aperture slits and transported to an experimental chamber. To avoid dissociation of incident cluster beams resulting from residual gas collisions, the base pressure of the beam line and experimental chamber was kept below $10^{-5} \mathrm{~Pa}$. The well-collimated beams were transmitted through a glass capillary mounted on a goniometer that allows for the adjustment of two independent capillary tilting angles $(\theta, \varphi)$ within $\pm 1.5^{\circ}$ relative to the beam direction. To avoid macroscopic charge-up at the capillary inlet due to beam irradiation, an aperture slit (with a $0.6-\mathrm{mm}$ diameter hole and smaller than the $0.8-\mathrm{mm}$ inner capillary diameter) was placed just before the capillary.

The tapered capillaries were manufactured from borosilicate glass tubes with outer and inner diameters of $2 \mathrm{~mm}$ and $0.8 \mathrm{~mm}$, respectively: they were produced by heating and stretching glass tubes. Special care was taken in cutting the tip of the stretched glass tube to produce a flat cross-sectional shape of the capillary exit. Figure 2 shows details of the capillary exit. The capillary outlet diameter was $13.7 \mu \mathrm{m}$. The capillary taper angle in the exit region was 0.1 degree or less. 
We measured the outgoing energies of the transmitted particles through a capillary by a solid state detector (SSD). To minimize energy loss of the transmitted particles in the SSD entrance window, we used a passivated implanted planar silicon (PIPS) detector with a window thickness of less than $500 \AA$ (CANBERRA, Model No. PD50-12-100AM). The detector was located at $9.1 \mathrm{~mm}$ downstream from the exit of the capillary. The SSD arrangement allows for detecting almost all transmitted particles through the capillary. Since PIPS detector active area is $50 \mathrm{~mm}^{2}$, the SSD acceptance angle (centered on the beam direction) is estimated to be about 47 degrees, which is much larger than the divergence angle of transmitted particles (as seen in Fig. 6). To avoid peak broadening of energy spectra due to cluster-impact radiation damage, we changed the SSD beam impact positions for each energy spectrum measurement. SSD-signal count rates were kept less than 20 counts per second.

Furthermore, we measured beam spot of transmitted particles with a solid-state nuclear track detector (CR-39), located $6.0 \mathrm{~mm}$ from the capillary exit, and obtained beam spot profiles by measuring etch pits for irradiation with about 600-800 particles. The chemical etching condition for the CR-39 detector was $7 \mathrm{~N} \mathrm{KOH}$ at $70{ }^{\circ} \mathrm{C}$ for 20 minutes.

By adjusting the capillary axis with respect to the beam direction, we measured energy spectra of the transmitted particles and determined the optimal capillary-axial adjustment for achieving maximum transmission fraction of the incident cluster ions without fragmentation.

\section{Results and discussion}

Figure 3 shows typical capillary-transmission energy spectra for $\mathrm{C}_{2}^{+}$projectiles at three different incident energies of $0.48,0.96$, and $1.92 \mathrm{MeV}$. The energy spectra of projectile beams before capillary-transmission are shown by broken lines. For incident cluster projectiles, the dissociation fraction is less than $5 \%$. The outgoing energy spectrum exhibits 
two peaks, namely a full energy peak corresponding to direct transmission of $\mathrm{C}_{2}$ ions without fragmentation (non-fragmentation process), and the other peak located at half of the incident energy corresponds to $\mathrm{C}_{1}$ fragments of $\mathrm{C}_{2}$ (fragmentation process). To understand the collision processes in the inner wall of capillary, our attention is directed to the energy spectra shape and relative peak intensity. One can see a low energy tail in the $\mathrm{C}_{1}$-fragment peaks, which becomes more significant with increasing the incident energy. The latter suggests an increase of scattered particle transmission in the capillary bulk with increasing incident energy. In contrast, no significant tails are observed in the peak corresponding to $\mathrm{C}_{2}$ detection. This evidently implies that the peak arises from the detection of non-fragmented $C_{2}$, and not from the mutual detection of two fragmented carbon atoms (pile-up effects).

Interestingly, the ratio of $\mathrm{C}_{2}$ to $\mathrm{C}_{1}$ peak intensities depends on the incident energy. To see the velocity dependence in more detail, Fig. 4 displays the fraction of the transmitted $\mathrm{C}_{2}{ }^{+}$ions relative to the total number of transmitted particles (hereafter referred to as the non-fragmentation fraction) as a function of the ion velocity. The non-fragmentation fraction significantly increases with decreasing ion velocity. This characteristics agrees qualitatively with the result by Stolterfoht et al. for molecular-beam transmission studies with $100 \mathrm{~nm}$ diameter PET nanocapillaries [11], who observed the transmission phenomenon without fragmentation for low-velocity $(1 \mathrm{keV}) \mathrm{H}_{2}{ }^{+}$and $\mathrm{H}_{3}{ }^{+}$molecular ions. Note that the outlet diameter or aspect capillary ratios in this work are completely different to those used in Ref. [11]. We have yet to perform a detailed correlation between the non-fragmentation fraction and capillary-diameters or aspect capillary ratios.

Next, we consider the cluster-size dependence of the transmission fraction. Figure 5 shows results of transmission energy spectra for $\mathrm{C}_{3}{ }^{+}$and $\mathrm{C}_{4}{ }^{+}$projectiles with the same velocity of 0.89 a.u.. For $\mathrm{C}_{3}{ }^{+}$projectiles, three peaks are observed: the higher energy peak corresponds to direct transmission $\mathrm{C}_{3}{ }^{+}$ions, and the two lower energy peaks correspond to dissociated $\mathrm{C}_{1}$ 
and $\mathrm{C}_{2}$ fragments. The low energy tail of the $\mathrm{C}_{2}$ peak is similar to that of $\mathrm{C}_{1}$, implying that the $\mathrm{C}_{2}$-peak is associated with pile-up effects; i.e., the mutual detection of two fragmented carbon atoms rather than non-dissociated $\mathrm{C}_{2}$ molecules. It is seen that the relative peak intensity of $\mathrm{C}_{3}$ is dominant compared with that of both fragments. A similar feature is observed for $\mathrm{C}_{4}^{+}$ projectiles. Comparing relative intensities among the fragment peaks, $\mathrm{C}_{1}$-fragment peak is dominant and decreases monotonically for larger fragment-sizes. This also provides evidence for pile-up detection of atomic fragments rather than molecular particles, as seen in the result for $\mathrm{C}_{3}$ projectiles.

Comparing non-fragmentation fractions for different cluster-sized projectiles with the same velocity, we observe that fractions are $62 \%, 68 \%$ and $55 \%$ for projectiles of $\mathrm{C}_{2}, \mathrm{C}_{3}$, and $\mathrm{C}_{4}$ ions, respectively, with $0.24 \mathrm{MeV} /$ atom energy (the corresponding velocity is 0.89 a.u.), indicating a well-known even-odd oscillation structure [12]. The latter structure is probably due to the dependence of non-fragmentation $C_{n}$ clusters on cluster stability even for capillary surface scattering. In other words, part of the direct transmission component is due to a contribution of grazing scattered molecular $\mathrm{C}_{n}$ with the inner capillary wall. This opens the possibility of using the capillary as a filter for molecular beams of special structure and alignment.

Figure 6 shows beam profile measurements of transmitted particles through a capillary for projectiles of atomic $\mathrm{C}_{1}{ }^{+}$and polyatomic cluster ions of $\mathrm{C}_{n}{ }^{+}(n=2-4)$ with the same energy of $0.24 \mathrm{MeV} /$ atoms; the profile results from measurements of etch pits of the nuclear track detector (CR-39). The profile consists of two components: a direct beam component and a scattered beam component. The direct beam component is mainly due to primary beam transmission without cluster-fragmentation; the spot size is almost the same as the capillary outlet diameter. The scattered beam component is due to dissociated fragment transmission, with lower intensity than the direct beam component. Atomic $\mathrm{C}_{1}^{+}$projectiles exhibit beam 
profiles that are similar to those of fast hydrogen ions [13]. To identify the type of transmitted particles detected in the scattered region, we performed detailed analyses of etch pit diameters of the CR-39 detector. Compared with the direct component, the scattered component pit diameter is smaller; this difference is attributed to radiation damaging effects or amount of energy deposition. From this result, we conclude that dissociated fragments mainly contribute to the scattering component.

We observe a significant difference between the scattered beam component angular distributions of atomic $\mathrm{C}_{1}$ and cluster $\mathrm{C}_{n}^{+}(n=2-4)$ projectiles. The cluster projectile distribution is narrower compared with atomic $\mathrm{C}_{1}$ projectiles, implying suppression of dissociated fragment transmission. This suppression is associated with the following factor, namely an increase of the internuclear separation of fragment-pairs by Coulomb repulsion. Therefore, the transmission suppression may occur for the outgoing fragments with an angular spread exceeding the acceptance angle of the capillary outlet. Hence, one can use a tapered capillary for cluster projectiles to reduce the angular spread of transmitted particles through the capillary.

\section{Summary}

We studied the capillary-transmission properties for $\mathrm{MeV}$-energy cluster beams of $\mathrm{C}_{n}^{+}$ $(n=2-4)$ ions. The capillary-transmission occurs via non-fragmentation and fragmentation processes. We found that the non-fragmented primary cluster fraction clearly correlates with projectile velocity: the fraction increases with decreasing velocity. This result indicates that cluster ions with low velocity contribute significantly to the non-fragmentation process. For different size projectiles of $\mathrm{C}_{n}{ }^{+}(n=2-4)$ ions with the same velocity, the fraction as a function of the cluster size shows an even-odd alternation that is probably due to the contribution of 
stable clusters surviving the grazing scattering process at the capillary surface. We also found that the transmission of cluster beams through a capillary have a smaller beam divergence compared with atomic beams; this property is advantageous for realizing beam-implantation into desired irradiation areas. The present cluster-microbeam method is applicable as a new probe for micro-ion-beam material analyses, including micro-SIMS or ion beam microfabrication.

\section{Acknowledgments}

We thank Professor T. Narusawa and Dr. T. Nebiki of Kochi University of Technology for instruction of capillary-preparation methods. We also thank staff members of the accelerator facility of the University of Tsukuba for their assistance in performing the experiments.

\section{References}

[1] T. Nebiki, T. Yamamoto, T. Narusawa, M.B.H. Breese, E.J. Teo, F. Watt, J. Vac. Sci. Technol. A 21(5) (2003) 1671

[2] T. Nebiki, D. Sekiba, H. Yonemura, M. Wilde, S. Ogura, H. Yamashita, M. Matsumoto, K. Fukutani, T. Okano, J. Kasagi, Y. Iwamura, T. Itoh, S. Kuribayashi, H. Matsuzaki, T. Narusawa, Nucl. Instrum. Meth. B 266 (2008) 1324

[3] T. Ikeda, Y. Kanai, T.M. Kojima, Y. Iwai, T. Kambara, Y. Yamazaki, M. Hoshino, T. Nebiki, T. Narusawa, Appl. Phys. Lett. 89 (2006) 163502

[4] B.S. Dassanayake, S. Das, R.J. Bereczky, K. Tőkési, J. Tanis, Phys. Rev. A 81 (2010) 020701(R)

[5] W. Wang, J. Chen, D.Y. Yu, B. Yang, Y.H. Wu, M.W. Zhang, F.F. Ruan, X.H. Cai, 
Phys. Scr. T144 (2011) 014023

[6] N. Oshima, Y. Iwai, T.M. Kojima, T. Ikeda, Y. Kanazawa, M. Hoshino, R. Suzuki, Y. Yamazaki, Materi. Sci. Forum 607 (2008) 263

[7] T. Nebiki, M.H. Kabir, T. Narusawa, Nucl. Instrum. Meth. B 249 (2006) 226

[8] M.J. Simon, M. Döbeli, A.M. Müller, H.-A. Synal, Nucl. Instrum. Meth. B 273 (2012) 237

[9] Y. Iwai, T. Ikeda, T.M. Kojima, Y. Yamazaki, T. Kobayashi, T. Nebiki, T. Narusawa, G.P. Pokhil, Appl. Phys. Lett. 92 (2008) 023509

[10] M. Kato, W. Meissl, K. Umezawa, T. Ikeda, Y. Yamazaki, Appl. Phys. Lett. 100 (2012) 193702

[11] N. Stolterfoht, R. Hellhammer, P. Sobocinski, Z.D. Pešić, J. Bundesmann, B. Sulik, M.B. Shah, K. Dunn, J. Pedregosa, R.W. McCullough, Nucl. Instr. and Meth. B 235 (2005) 460

[12] E. A. Rohlfing, D. M. Cox, A. Kaldor, J. Chem. Phys. 81 (1984) 3322

[13] S. Jaiyen, N. Chankow, J. Hasegawa, Y. Oguri, Nucl. Instrum. Meth. B 271 (2012) 13

\section{Figure Captions}

Fig. 1 Schematic diagram of the experimental setup.

Fig. 2 Distributions of the capillary taper angle as a function of capillary distance from the exit. Photographs show the cross-sectional shape of the capillary exit (left) and the side view of the capillary near the exit (right). 
Fig. 3 Energy spectra of transmitted particles through the capillary with $13.7 \mu \mathrm{m}$ diameter for $\mathrm{C}_{2}{ }^{+}$projectiles with the energies of 0.240 .96 , and $1.92 \mathrm{MeV}$ (solid lines). The broken line corresponds to energy spectra of incident beams without the capillary.

Fig. 4 Relative ratio of the $\mathrm{C}_{2}$-transmission (number of the transmitted $\mathrm{C}_{2}$ ions divided by the total number of the transmitted particles ) as a function of projectile velocity.

Fig. 5 Energy spectra of transmitted particles for $\mathrm{C}_{3}{ }^{+}$and $\mathrm{C}_{4}{ }^{+}$projectile ions with the same velocity of 0.89 a.u. (solid lines). The broken line corresponds to incident beam energy spectra.

Fig. 6 Two-dimensional distributions of the fraction of dissociated fragments transmitted through the capillary of $13.7 \mu \mathrm{m}$ diameter for four different projectile species with the same velocity (0.89 a.u.). The bar graph shows a fraction of the scattering component as a function of distance from the beam spot center. 


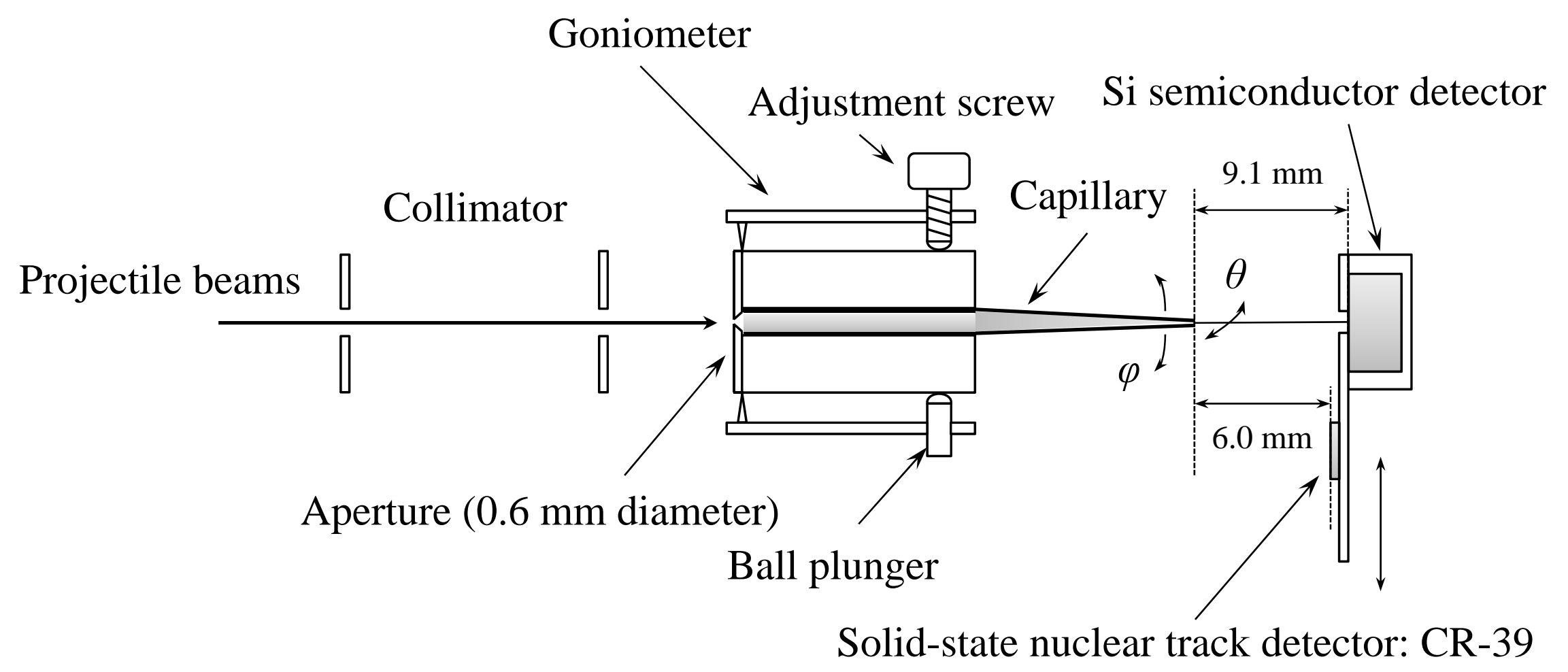

Fig. 1 Tsuchida et al . 


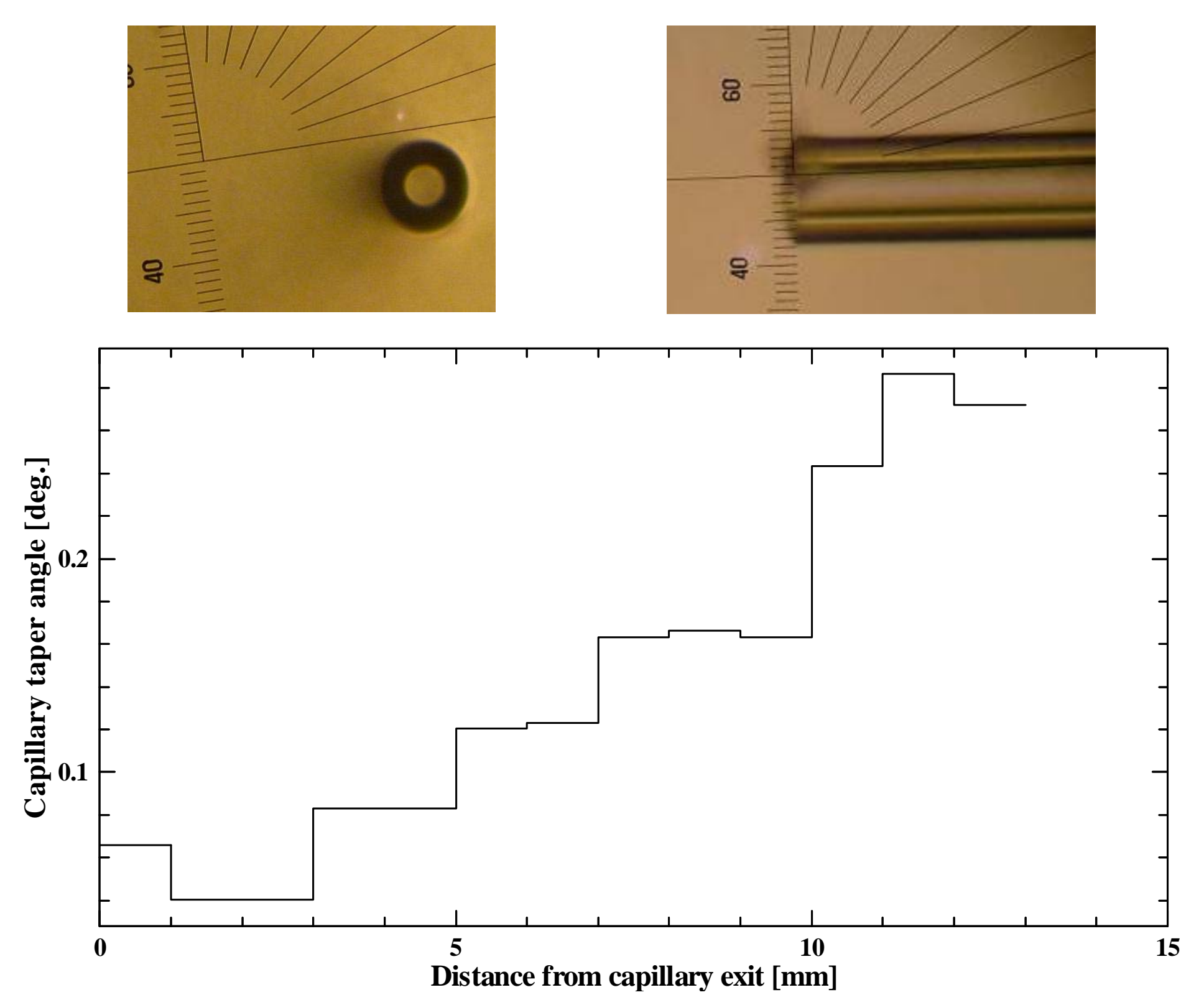

Fig. 2 Tsuchida et al. 


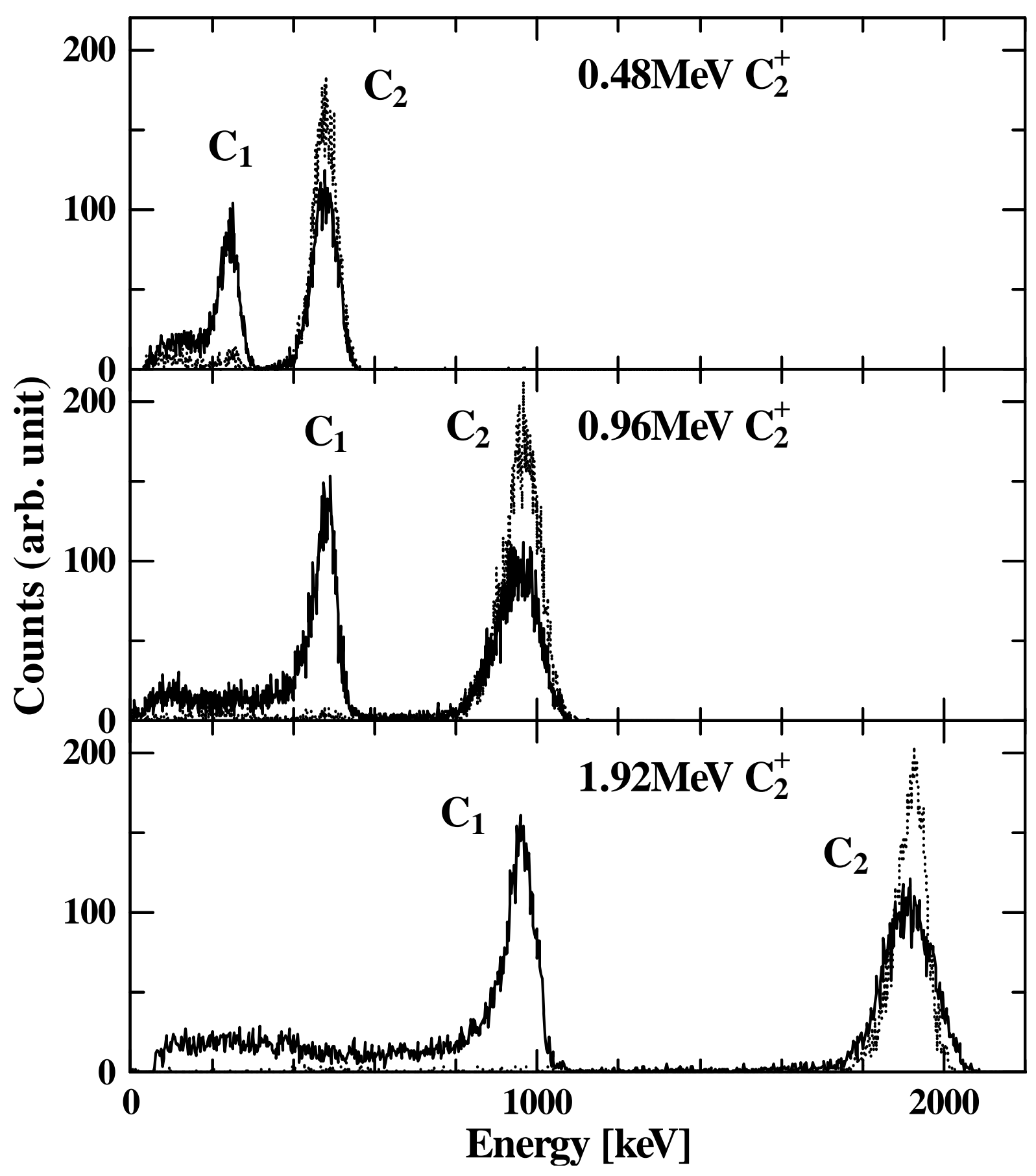

Fig. 3 Tsuchida et al. 


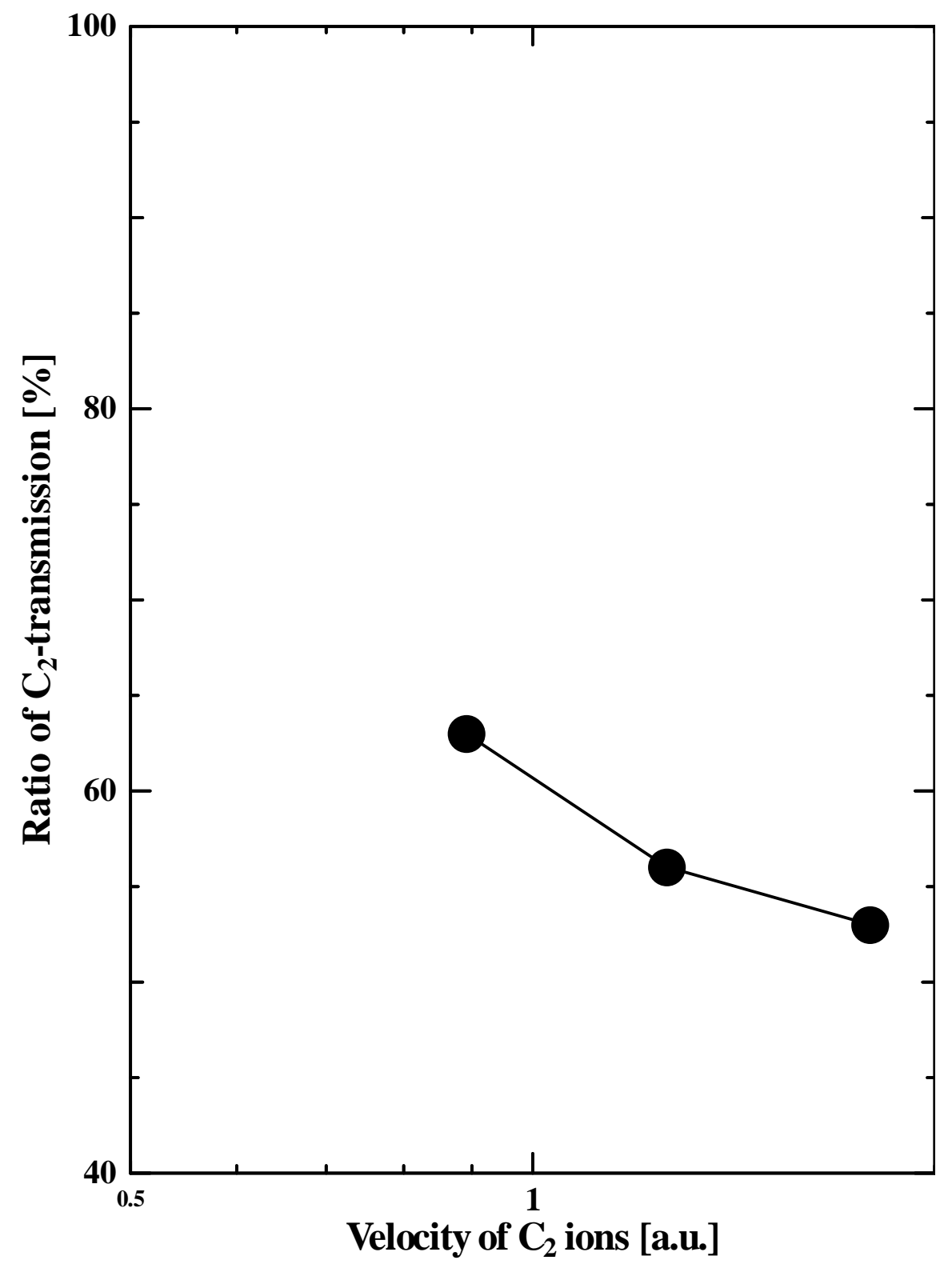

Fig. 4 Tsuchida et al. 


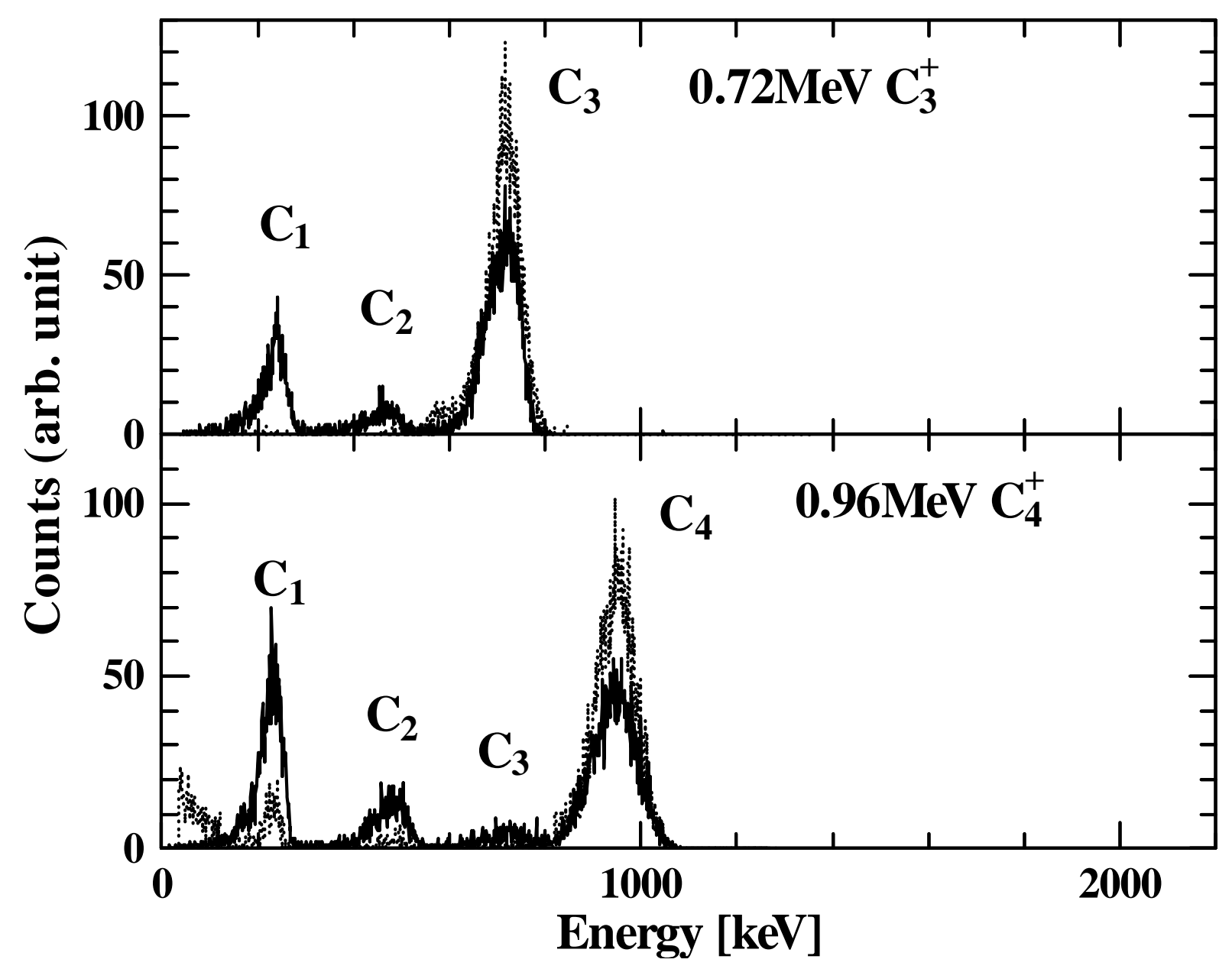

Fig. 5 Tsuchida et al. 

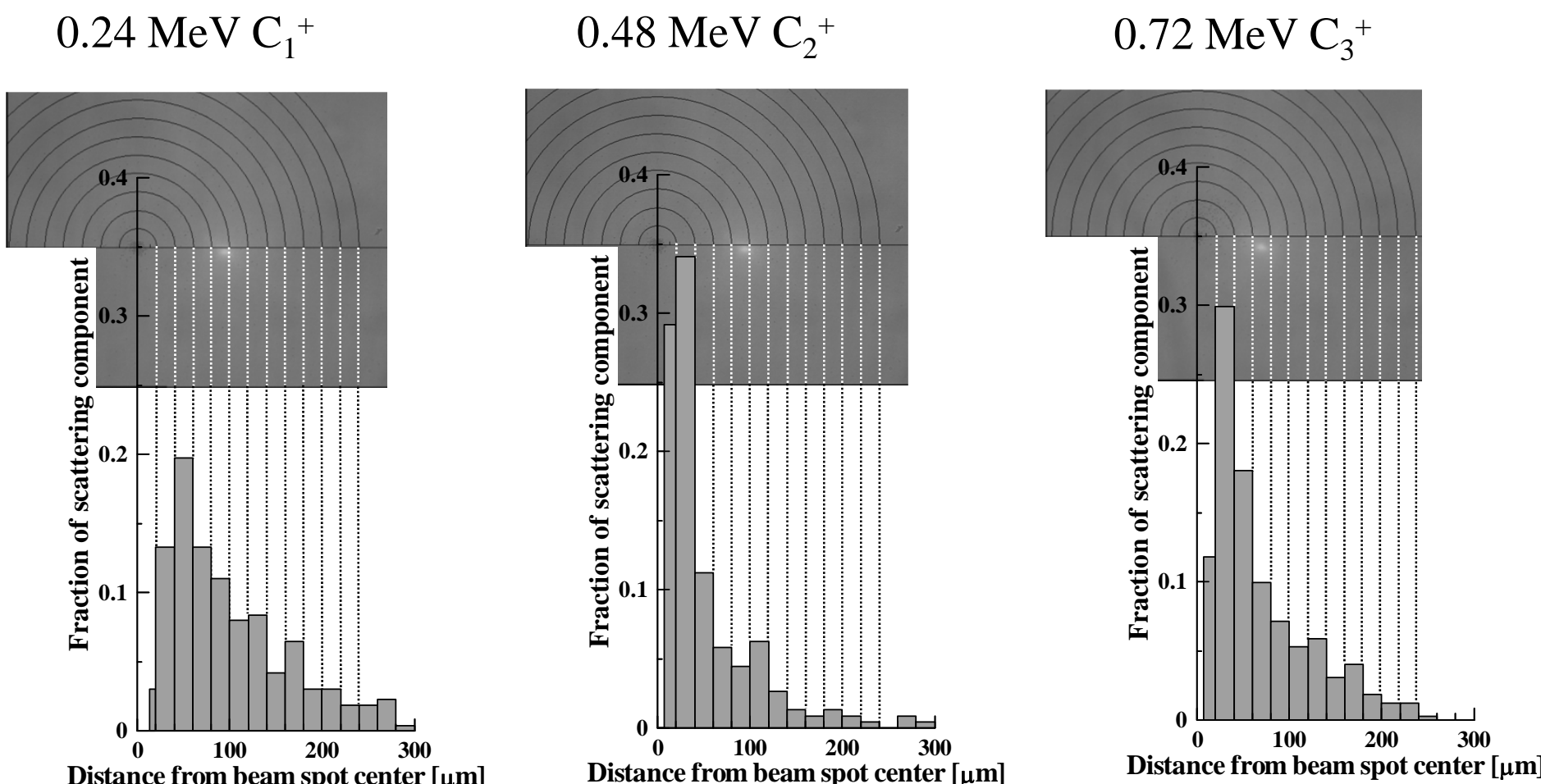

$0.96 \mathrm{MeV} \mathrm{C}_{4}^{+}$

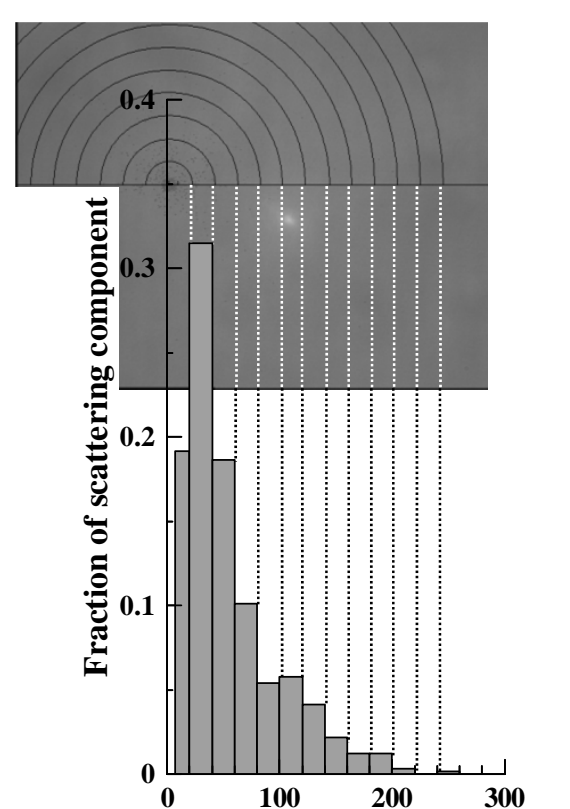

Distance from beam spot center $[\mu \mathrm{m}]$

Fig. 6 Tsuchida et al . 